\title{
Morphological and physiological effects of two different light sources on in vitro multiplication of chestnut and prickled broom
}

\author{
J. C. Gonçalves ${ }^{1,2 *}$, A. $\operatorname{Skec}^{2,3}$, A. Krnjac ${ }^{2,3}$, T. Delgado ${ }^{1}$, D. Frazão ${ }^{1}$, N. Farinha ${ }^{1}$, J. \\ Domingues $^{1}$ and T. Coelho ${ }^{1,2}$
}

${ }^{1}$ Plant Biotechnology Center of Beira Interior, Quinta Senhora de Mércules, 6001-909 Castelo Branco, Portugal; ${ }^{2}$ Polytechnic Institute of Castelo Branco, School of Agriculture, Quinta Senhora de Mércules, Apartado 119, 6001-909 Castelo Branco, Portugal; ${ }^{3}$ Krizevci College of Agriculture, Krizevci, Croatia.

*E-mail: jcgoncalves@ipcb.pt

\begin{abstract}
The morphology and physiology of plants grown in vitro are regulated by various environmental factors such as light, temperature, humidity and carbon dioxide. Light, in its various parameters such as spectral quality, photon flux and photoperiod, is an important factor among these and generally influences the overall growth and development of plants in vitro. Light sources commonly used for in vitro plant culture are fluorescent lamps, although some research and commercial laboratories also use sodium metal or incandescent metal halide lamps. The spectral of those lamps range from 350 to $750 \mathrm{~nm}$ and contain blend lights that differentially affect in vitro plant growth. Recently, light emitting diodes (LEDs) have been developed and are nowadays used as an alternative source of fluorescent light for the development of plants both in greenhouses and in vitro culture rooms. In order to test the influence of these two types of light during multiplication phase, a multiplicity experiment was carried out using two species: a chestnut hybrid (Castanea sativa x C. crenata), clone M2 and the prickled broom (Pterospartum tridentatum (L.) Willk.), Malcata ecotype. Two types of light were tested, "cool white" fluorescent light and LED light with blue and red colors. It was found that LED illumination exerted a significant positive effect on the differentiation of shoots in the case of prickled broom (5.6 instead of 4.9 split shoots under fluorescent light). In the parameters of shoot length, we found that this type of lighting favored the elongation in both species. Regarding the parameters of fresh and dry weight, LED lighting caused a greater effect on the chestnut than on prickled broom. Some physiological parameters (chlorophylls, carotenoids and proteins) were also analyzed and showed to be influenced by the type of light during the development of microplants.
\end{abstract}

Keywords: Chestnut; fluorescent light; in vitro multiplication; LEDs; prickled broom.

VII International Symposium on Production and Establishment of Micropropagated Plants.

Lavras, Minas Gerais (Brazil); April 24-28, 2017; \#PEMP2017 